EGU21-2203, updated on 27 May 2021

https://doi.org/10.5194/egusphere-egu21-2203

EGU General Assembly 2021

(c) Author(s) 2021. This work is distributed under

the Creative Commons Attribution 4.0 License.

\title{
A licensing system for planetary geospatial data for the GMAP project
}

\author{
Alessandro Frigeri ${ }^{1}$, Angelo Pio Rossi ${ }^{2}$, Andrea Nass ${ }^{3}$, and Matteo Massironi ${ }^{4}$ \\ ${ }^{1}$ Istituto Nazionale di Astrofisica, Istituto di Astrofisica e Planetologia Spaziali, Roma, Italy (alessandro.frigeri@inaf.it) \\ ${ }^{2}$ Jacobs University, Bremen, Germany \\ ${ }^{3}$ Deutsches Zentrum für Luft- und Raumfahrt (DLR), Berlin, Germany \\ ${ }^{4}$ Dipartimento di Geoscienze, Università di Padova, Padova, Italy
}

The Geologic Mapping of Planetary Bodies (GMAP) project Integrates partners and outputs from two projects previously funded by the EU through Horizon 2020 (UPWARDS and PLANMAP) to deliver tools and services for geological mapping of any Solar System body. Started in 2020, GMAP is developing an infrastructure to support future European missions in developing orbital acquisition strategies, rover deployment and traverses, and human exploration programs. Part of GMAP deals with the study of current procedures for publishing planetary maps, and the development of new ones. Since the Apollo era, geologic maps of the Moon and bodies of the solar system have been produced and disseminated by the United Stated Geologic Survey, Astrogeology Program, funded by NASA. Being both USGS and NASA governmental organization of the same country, the coordination and the production of planetary maps followed a straightforward development from the beginning to the digital-era. In their digital form, the US maps have been made available under the public domain.

At the international level, every country has its own space agency or office but no public domain planetary maps have been systematically made available yet in re-usable formats outside the US. In Europe, space programs can be either promoted by European Space Agency or by any one of the participating states' space agencies, which is not necessarily an EU member. This is not ideal for a coordinated work for geoscientific mapping or dissemination of unified planetary mapping products.

Within GMAP we are surveying existing licensing models, looking for a licensing system that guarantees dissemination and the re-use of planetary mapping, the maximum compatibility with the existing dataset and protects original creator rights. We will report the status of our study and plans for the future. 